\title{
A Categorized Resource Sharing Mechanism for Device-to-Device Communications in Cellular Networks
}

\author{
Jie Chen, ${ }^{1}$ Chang Liu, ${ }^{2}$ Husheng Li, ${ }^{3}$ Xulong Li, ${ }^{1}$ and Shaoqian Li ${ }^{1}$ \\ ${ }^{1}$ University of Electronic Science and Technology of China, Chengdu, China \\ ${ }^{2}$ Dalian University of Technology, Dalian, China \\ ${ }^{3}$ Department of Electrical Engineering and Computer Science, The University of Tennessee, Knoxville, TN, USA \\ Correspondence should be addressed to Jie Chen; jiechenuestc@163.com
}

Received 12 July 2016; Revised 30 September 2016; Accepted 24 October 2016

Academic Editor: Piotr Zwierzykowski

Copyright (C) 2016 Jie Chen et al. This is an open access article distributed under the Creative Commons Attribution License, which permits unrestricted use, distribution, and reproduction in any medium, provided the original work is properly cited.

Device-to-Device (D2D) communications are considered one of the key technologies for 5G wireless communication systems. In this paper, a resource sharing mechanism, which applies different policies for different cases (thus being categorized), is proposed. In this scheme, all D2D pairs are divided into three groups by comparing the minimum transmit power with the maximum transmit power of each cellular UE. The proposed mechanism enables multiple D2D pairs in the second group to share the resource with cellular user equipment (UE) simultaneously, by adjusting the transmit powers of these D2D transmitters. At the same time, D2D pairs in the first group and the third group share resource with cellular UE based on the transmit power minimization principle. Simulation results show that the proposed scheme can achieve relatively higher network throughput and lower transmit power consumption of the D2D system.

\section{Introduction}

The increasing demand for higher data rates within local area and the gradually increasing spectrum congestion have triggered research activities on improving the spectral efficiency and interference management. In recent years, D2D (Deviceto-Device) communications have gained much attention [1, 2]. D2D communications enhance the spectral efficiency by spatially reusing radio resource and prolong the battery life of user equipment (UE) by reducing the transmission power. Due to these advantages, the D2D communications have been actively discussed in standardization bodies for the next generation cellular systems such as Long Term EvolutionAdvanced (LTE-A) [3].

However, D2D links may yield significant interference to the communication system. Therefore, resource management scheme, which supports the resource reuse by taking the intracell interference into account, has a great impact on the overall network performance. So far, major efforts are to be aimed at interference control through resource sharing mode selection [4], power control [5-7], and resource allocation [8-10]. The previous works [5-7] are mainly concentrated on the power control in a specific resource block without the consideration of resource allocation, while the previous works in [8-10] have focused on the case that only one D2D link can share resource with one cellular link. Additionally, in [11], the authors study joint channel and power allocation to improve the energy efficiency of user equipment. In [1215], the authors design the power-based resources allocation scheme and achieved relatively better performance, while they do not consider the factor of geography distribution, which may affect the power seriously. In [16], the authors proposed an interference-aware graph based resource sharing algorithm that can effectively obtain the near-optimal resource assignment solutions at the base station but with low computational complexity. In [17], a resource allocation scheme based on a column generation method is proposed. In [18], the authors aimed to optimize resource sharing for D2D communication to better utilize uplink resources in a multiuser cellular system with guaranteed quality of normal cellular communications.

In this paper, to further improve spectrum utilization and system capacity, a resource sharing method, which enables multiple D2D links to share resource with cellular UE 
simultaneously, is proposed. Firstly, the minimum transmit power of a D2D transmitter is calculated by the required minimum Signal-to-Interference-plus-Noise Ratio (SINR) and the interference from cellular UE which shares the resource with the D2D link. Secondly, based on the interference threshold of eNodeB, the maximum transmit power of a D2D transmitter is calculated. Thirdly, by comparing the minimum transmit power with the maximum one on the resource of each cellular UE, a set of cellular UE devices that can share resource with the D2D link is attained. Then, the D2D pairs are divided into three groups according to the comparison result.

Finally, when many D2D links can only share resource with some special cellular UE or the number of D2D links is larger than the number of cellular UE devices, the transmit powers of these $\mathrm{D} 2 \mathrm{D}$ transmitters are adjusted to ensure that the cumulative interference to eNodeB is below a threshold; and the minimum SINR value of each D2D link is met as well. After that, they can share resource with the cellular UE simultaneously. Simulation results show that the proposed scheme can achieve a higher network capacity and lower transmit power consumption of the D2D system.

Then, we can summarize the main contributions in the following:

(i) Different from the existing works, we analyze the resource sharing from the feasibility of transmit power and design the power bound principle by the requirement of minimum SINR and maximum interference, which provides a novel power management scheme.

(ii) According to the different geometry distribution (distances of various nodes in the system), the resources are divided into 3 groups based on the power bound principle, thus fully utilizing the UE resources.

(iii) Taking advantage of the adjustable transmit power of D2D, the proposed mechanism enables multiple D2D pairs in the second group to share the resource with cellular UE simultaneously. In addition, D2D pairs in the first group and the third group can share resource with cellular UE based on the transmit power minimization principle, which further improves the power efficiency.

The remainder of this paper is organized as follows. The next section describes the system model. The resource sharing method between cellular UE and D2D pairs is presented in Section 3. The performance of the proposed method is evaluated in Section 4 and the paper is concluded with Section 5.

\section{System Model}

Considering an OFDMA based cellular network, which is frequency division duplex (FDD), and concentrating on a single cell served by eNodeB as depicted in Figure 1, it is assumed that the eNodeB knows the path-loss components between any two UE devices and between any UE and the eNodeB, based on the locations of the UE or the average

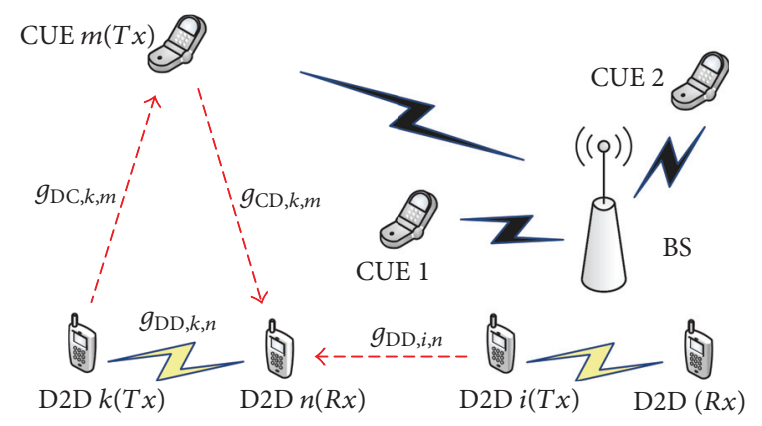

FIGURE 1: Device-to-Device communication scenarios in a cellular network.

channel qualities [19]. In the cell, there are $m$ cellular UE devices, where $m=1, \ldots, M$. In addition to $m$ cellular UE devices, there are $k$ pairs of D2D UE devices which communicate directly with each other, where $k=1, \ldots, K$. Consider the notion that D2D UE devices only share uplink resource with cellular UE. Furthermore, it is also assumed that exclusive resources are reserved for $N$ pairs of D2D UE devices.

In this work, we focus on the factor of path loss due to the different distance between the D2D pairs and UE. The different distances lead to the different channel gains and thus can further affect the transmit power. According to Figure 1, $g_{\mathrm{DD}, k, n}$ represents the channel gain between D2D $k$ and D2D $i$, while $g_{\mathrm{CD}, m, k}$ signifies the channel gain between cellular UE $m$ and $\mathrm{D} 2 \mathrm{D} k$, and $g_{\mathrm{DC}, k, m}$ denotes the channel gain between D2D $k$ and cellular UE $m$

\section{The Proposed Resource Sharing Mechanism}

3.1. Conditions for Sharing Resource between a Cellular Link and D2D Links. We define $u$ as a set of D2D links, which can share uplink resources with the cellular UE $m$ if and only if the following condition is met:

$$
\operatorname{SINR}_{k}=\frac{P_{k} g_{\mathrm{DD}, k, n}}{N_{k}+I_{m, k}+\sum_{i \in u, i \neq k} P_{i} g_{\mathrm{DD}, i, k}} \geq T_{0},
$$

$\forall k \in u$,

$$
\begin{aligned}
\sum_{k \in \mathcal{u}} P_{k} g_{\mathrm{DC}, k, m} & \leq I_{0}, \\
I_{m, k} & =P_{m} g_{\mathrm{CD}, m, k} .
\end{aligned}
$$

Here, $T_{0}$ and $I_{0}$ are defined as the minimal SINR value of D2D $k$ and the interference threshold value of cellular UE, respectively. $P_{k}$ and $P_{m}$ denote the transmit power of D2D link $k$ and transmit power of cellular UE $m$, respectively.

\subsection{Resource Sharing and Power Adjustment}

3.2.1. Acquiring Transmit Power Matrix. We define a $K \times M$ matrix $X$, of which the $(k, m)$ th element $x_{k, m}$ denotes the 


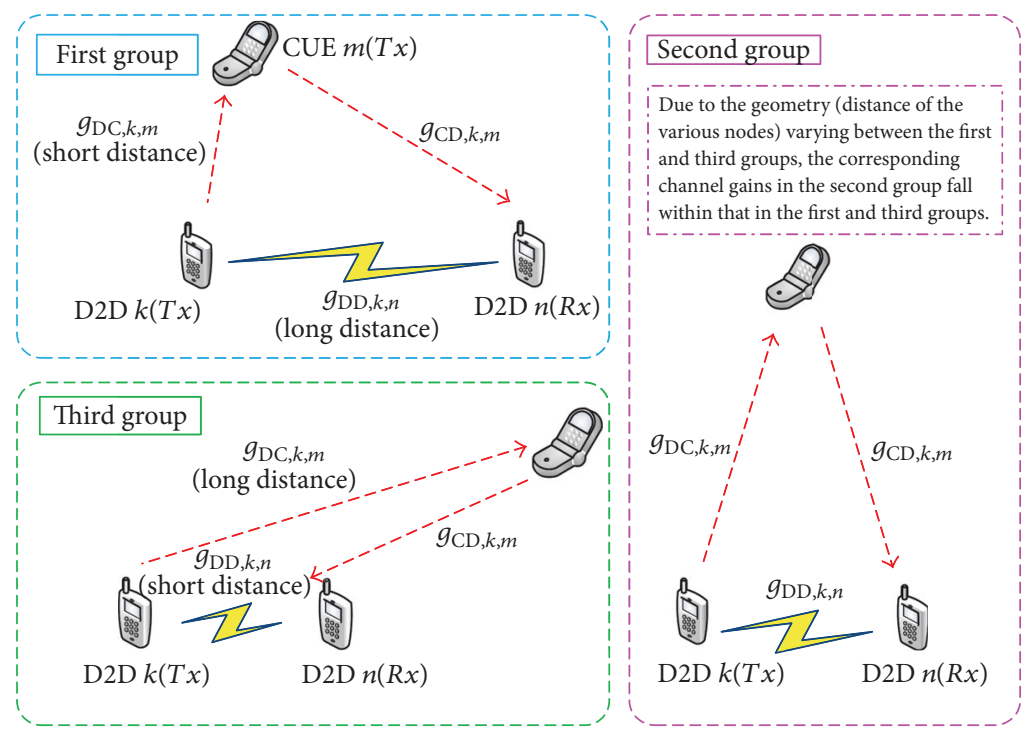

FIGURE 2: Grouping illustration for a cellular network.

minimum transmitter power of D2D $k$ that shared resources with cellular UE $m$, where $x_{k, m}$ can be calculated by (4). We define a $K \times M$ matrix $Y$, of which the $(k, m)$ th element $y_{k, m}$ means the maximum transmitter power of D2D $k$ that shared resources with cellular UE $m$, where $y_{k, m}$ can be calculated by (5). Hence,

$$
\begin{aligned}
\operatorname{SINR}_{k} & =\frac{x_{k, m} g_{\mathrm{DD}, k, m}}{N_{k}+P_{m} g_{\mathrm{CD}, m, k}} \geq T_{0} \\
I_{k} & =y_{k, m} g_{\mathrm{DB}, k, m} \leq I_{0} .
\end{aligned}
$$

Here, $T_{0}$ and $I_{0}$ are defined as the minimal SINR value of D2D $k$ and the interference threshold value of cellular UE, respectively.

3.2.2. Grouping $D 2 D U E$. According to the condition for sharing resource between the cellular link $m$ and D2D link $k, k$ cannot share resource with $m$ if $x_{k, m}>y_{k, m}$. Then, D2D pairs are divided into the following three groups by comparing each element of matrix $X$ and matrix $Y$ :

(i) First group (if all $x_{k, m}$ are more than all $y_{k, m}$ ): D2D pairs in the first group cannot share resource with any cellular UE.

(ii) Second group (if part of $x_{k, m}$ are more than part of $\left.y_{k, m}\right)$ : D2D pairs in the second group can share resource with some cellular UE.

(iii) Third group (if all $x_{k, m}$ are less than all $y_{k, m}$ ): D2D pairs in the third group can share resource with all cellular UE.

For further understanding, according to the geography distribution (distances of the various nodes), we then draw Figure 2 to address the categorization problem. It is shown that if the distance between D2D $k(T x)$ and CUE $m(T x)$ is short and the distance between D2D $k(T x)$ and D2D $n(R x)$ is long, then the channel gain $g_{\mathrm{DC}, k, m}$ turns to large value and $g_{\mathrm{DD}, k, n}$ turns to small value. In this case, the corresponding minimum transmit power in (4) may be larger than the maximum transmit power in (5), which makes the first group and D2D pairs unable to share resource with any cellular UE. Otherwise, the corresponding minimum transmit power in (4) may be smaller than the maximum transmit power in (5) and this situation belongs to the third group. Hence, as for the second group, due to the geometry (distance of the various nodes) varying between the first and third groups, the corresponding channel gains in the second group fall within that in the first and third groups.

Now, the resource sharing model, which enables multiple D2D UE devices to reuse the resource of cellular UE and minimizes the total transmit power of D2D system, is presented. We concentrate on resource sharing between cellular UE and D2D links in the second group. We construct an $L$-by$M$ matrix $P$ by selecting rows corresponding to the $\mathrm{D} 2 \mathrm{D}$ links in the second group from matrix $X$, that is, selecting the $i$ th row from matrix $X$ if D2D link belongs to the second group. Similarly, we construct an $L$-by- $M$ matrix $P$ by selecting rows from matrix $Y$. $L$ denotes the number of D2D pairs in the second group. Then, two algorithms are provided for seeking the minimum transmit power value in matrix $P$ under the following two cases.

Case $1(L<M)$. In Algorithm $1, X$ and $Y$ denote the row and the column of $P$. Lines (2)-(9) in Algorithm 1 are used for seeking the minimum transmit power value $p$ in matrix $P$, then marking the row and column that $p$ belongs to, and continuing searching for the minimum transmit power value in the unmarked rows and columns in matrix $P$; the algorithm repeats the above operation about $L$ times, thus obtaining $L$ minimum transmit power values. Lines (10)(18) are explained as follows: if each of these transmit power 


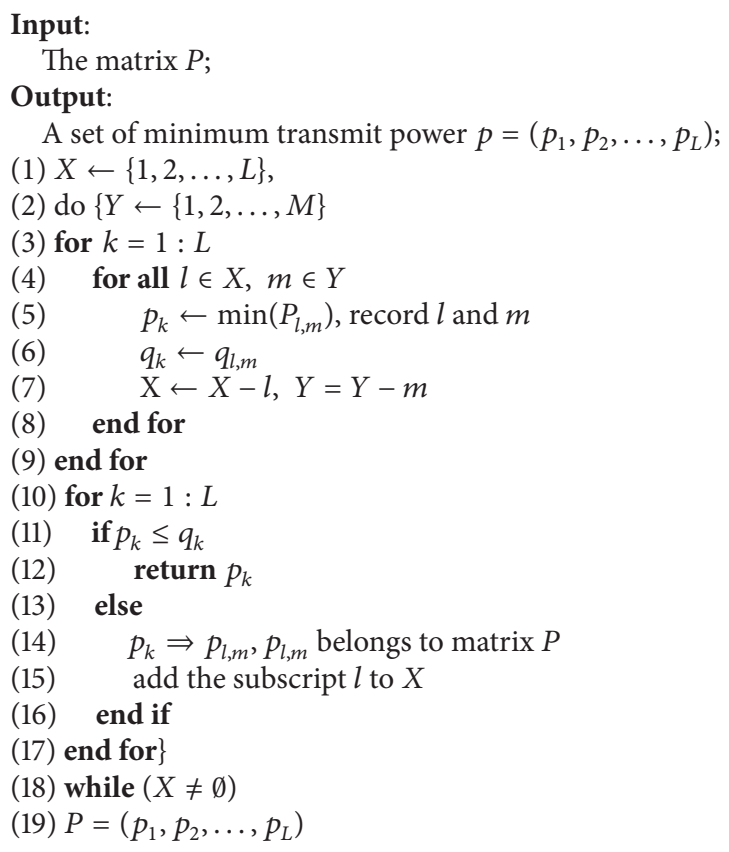

Algorithm 1: Seeking the minimum transmit power value.

values is less than the corresponding maximum transmit power value, the algorithm returns them. If some of them are larger than the corresponding maximum transmit power, Algorithm 1 continues searching for the minimum transmit power of these D2D links according to lines (2)-(9) until each of them is less than the corresponding maximum transmit power. In line (19), $p_{k} \Rightarrow p_{l, m}$ means that $p_{k}$ is the $(l, m)$ th element of matrix $P$.

Case $2(L>M)$. In Algorithm 2, first, it searches for $M$ minimum transmit power values from matrix $P$ by calling Algorithm 1. Then, it updates all the columns in matrix $P$ to unmarked and searches for the minimum transmit power values of the other $L-M$ D2D links by calling Algorithm 1 . When a feasible set $p$ is obtained, a set of transmit power values in matrix $X$ can be also attained by $p_{k} \Rightarrow p_{l, m} \Rightarrow$ $x_{k, m}$. If these transmit power values $\left(x_{k, m}\right)$ belong to different rows and different columns, the D2D links share resource with cellular UE by the subscript of the minimum transmit power; that is, $x_{i, j}$ means that D2D link $i$ shares resource with cellular UE $j$. However, some minimum transmit power values belong to the same column, which means that multiple D2D links compete for the resource of cellular UE.

Thus, according to (1) and (3), the algorithm adjusts the transmitter power of these D2D links, such that they can share resource with the cellular UE when (2) holds. Otherwise, a D2D link, of which the transmit power value is minimal to share resource with the cellular UE, is selected.

As mentioned above, the target of the resource sharing method is not only to improve the system capacity but also to minimize the transmit power of $\mathrm{D} 2 \mathrm{D}$ system. In order to
TABLE 1: Simulation parameters.

\begin{tabular}{lc}
\hline Cell radius & $500 \mathrm{~m}$ \\
Maximal distance between one D2D pair & $50 \mathrm{~m}$ \\
Number of cellular users & 25 \\
Number of D2D pairs & 25 \\
Bandwidth per RB $W_{\mathrm{RB}}$ & $180 \mathrm{kHz}$ \\
Path-loss model & $35.3+37.6 \log _{10} d \mathrm{~dB}$ \\
Target bit error rate BER ${ }^{1}$ & 0.01 \\
The probability threshold $\theta$ & 0.01 \\
Power level of thermal noise $N_{0}$ & $-174 \mathrm{dBm} / \mathrm{Hz}$ \\
The mean $\mu_{\varphi}$ of multipath fading & $1 \mathrm{~dB}$ \\
The standard deviation $\sigma_{n}$ of shadowing & $8 \mathrm{~dB}$ \\
\hline
\end{tabular}

${ }^{1}$ Note that BER is an important effect factor on the throughput for systems; that is, the larger the BER, the smaller the achievable throughput. Thus, we add it to the simulation parameters for the calculation of achievable throughput.

minimize the total transmit power of D2D system, D2D links in the second group and the third group share resource with cellular UE based on the power minimization principle. The exclusive resources prefer to be allocated to the D2D UE of smaller transmit power in the first group, while each of the remaining cellular UE devices prefers to share resource with the D2D UE of smaller transmit power in the third group.

\section{Performance Analysis}

In this section, the performance of the proposed resource sharing mechanism is evaluated. First, the simulation parameters are set and then the simulation results are presented and analyzed. All the simulations are operated under the MATLAB environment.

4.1. Simulation Setup. There are $M$ cellular UE devices and $K \mathrm{D} 2 \mathrm{D}$ pairs within a single circular cell with the radius of $500 \mathrm{~m}$. It is assumed that the bandwidth of an RB is $W_{\mathrm{RB}}=180 \mathrm{kHZ}$, and the noise spectral density is $N_{0}=$ $-174 \mathrm{dBm} / \mathrm{Hz}$. The path loss, shadowing, and Rayleigh fading are considered. Based on LTE system models [19], the pathloss model is $z=35.3+37.6 \log _{10} d(\mathrm{~dB})$, where $d$ is the distance between the transmitter and the receiver. According to [20], it is assumed that the shadowing components of all links are i.i.d., and the shadowing component $\eta$ follows a log-normal distribution with zero mean and standard deviation $\sigma_{n}, \sigma_{n}=8 \mathrm{~dB}$. It is also assumed that the multipath components of all links are independent of each other, and all of them are exponentially distributed with the same mean of $\mu_{\varphi}, \mu_{\varphi}=1$. The minimal SINR threshold value of each D2D pair for normal communication is $T_{0}=0 \mathrm{~dB}$, and the transmit power of each cellular UE is $20 \mathrm{dBm}$. We consider the number of D2D pairs and the interference threshold value of eNodeB $I_{0}$ as variables of the simulation. Simulation parameters are summarized in Table 1.

4.2. Simulation Results. The simulation results are plotted in Figures 3-8. Before discussing the results, we shortly describe 


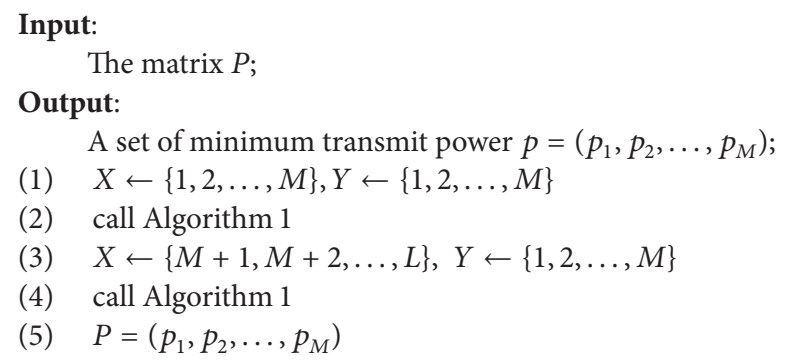

Algorithm 2: Seeking the minimum transmit power value.
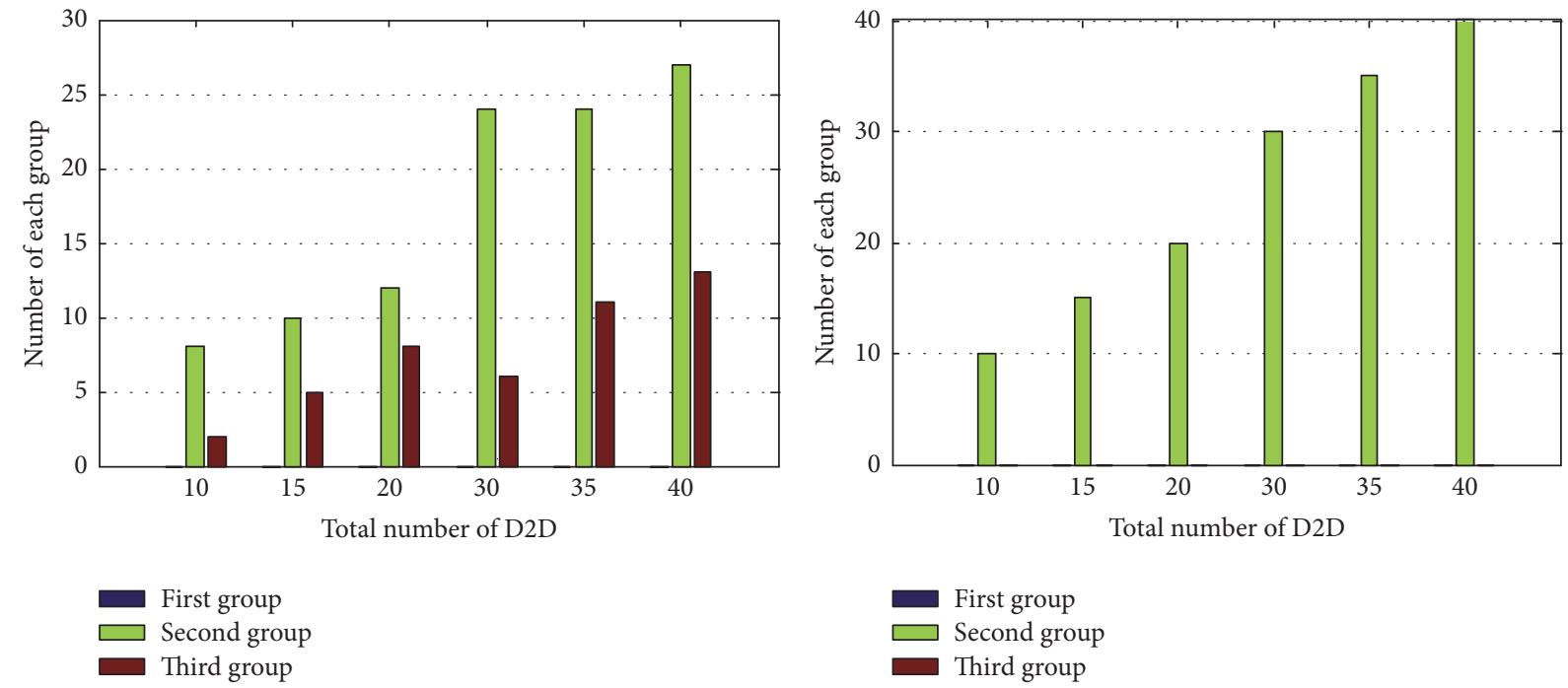

(a) $I_{0}=-100 \mathrm{~dB}$

(b) $I_{0}=-120 \mathrm{~dB}$

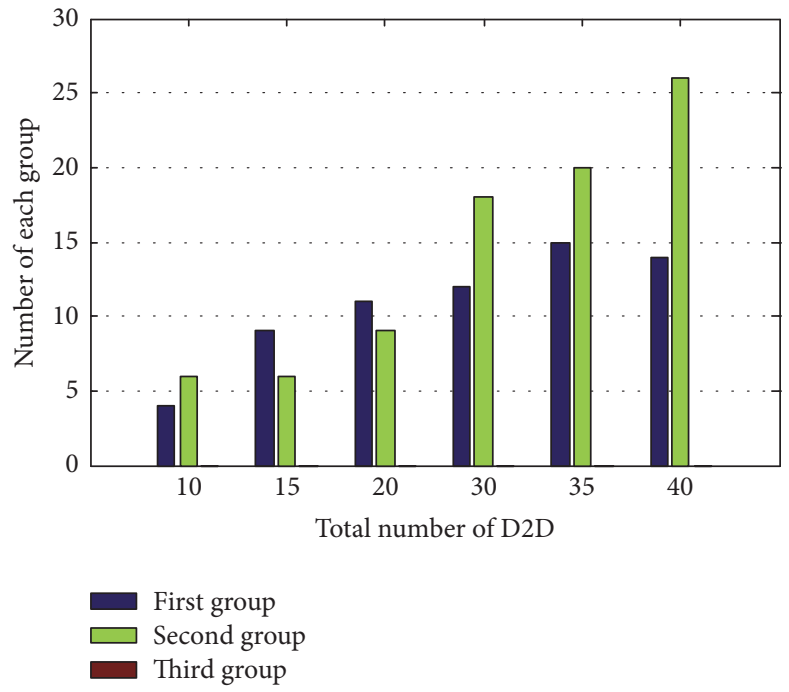

(c) $I_{0}=-130 \mathrm{~dB}$

FIGURE 3: The number of D2D pairs in each group. 


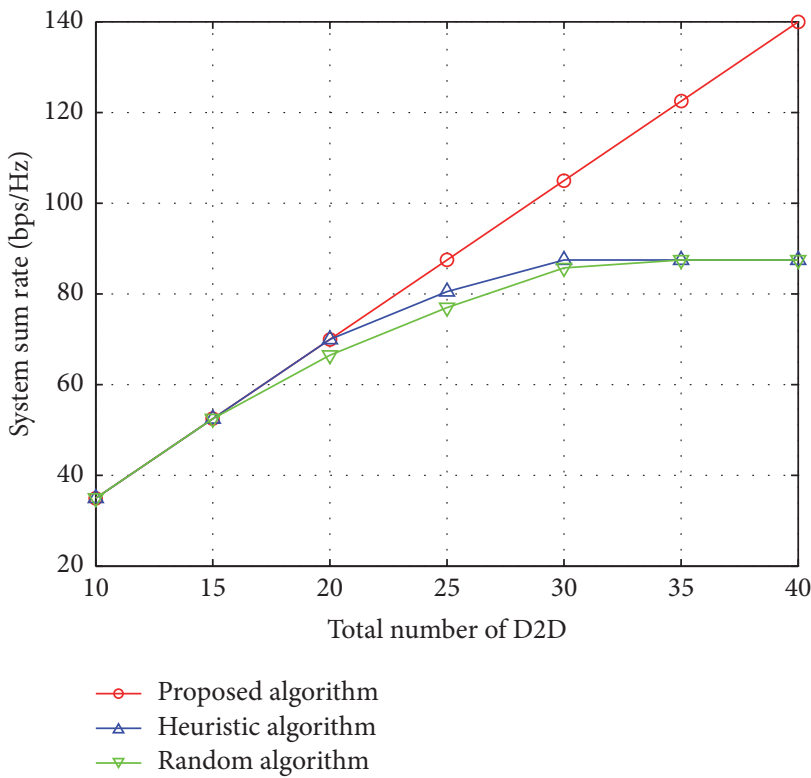

(a) $I_{0}=-100 \mathrm{~dB}$

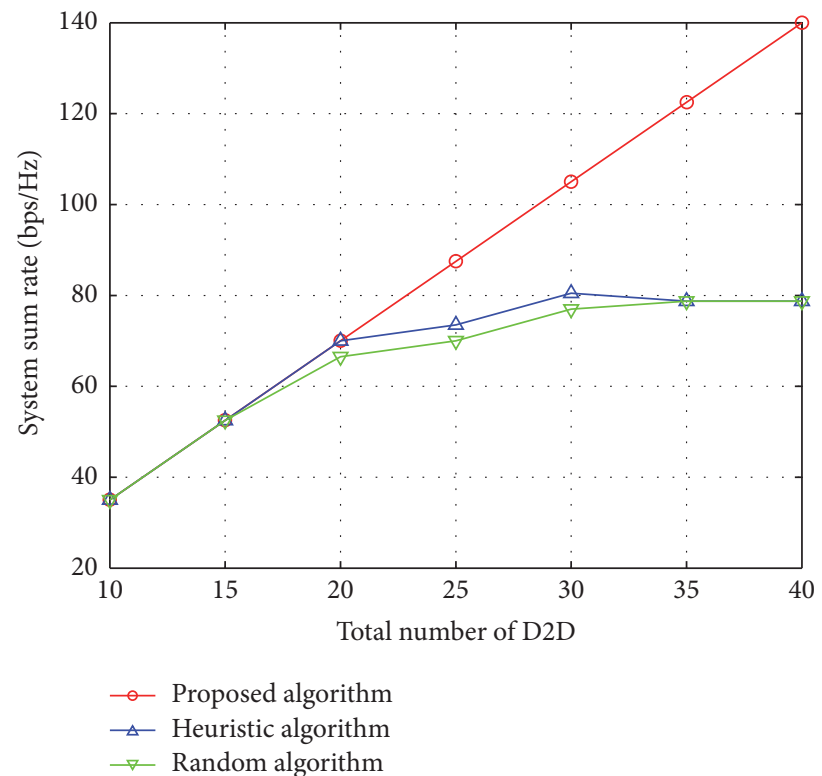

(b) $I_{0}=-120 \mathrm{~dB}$

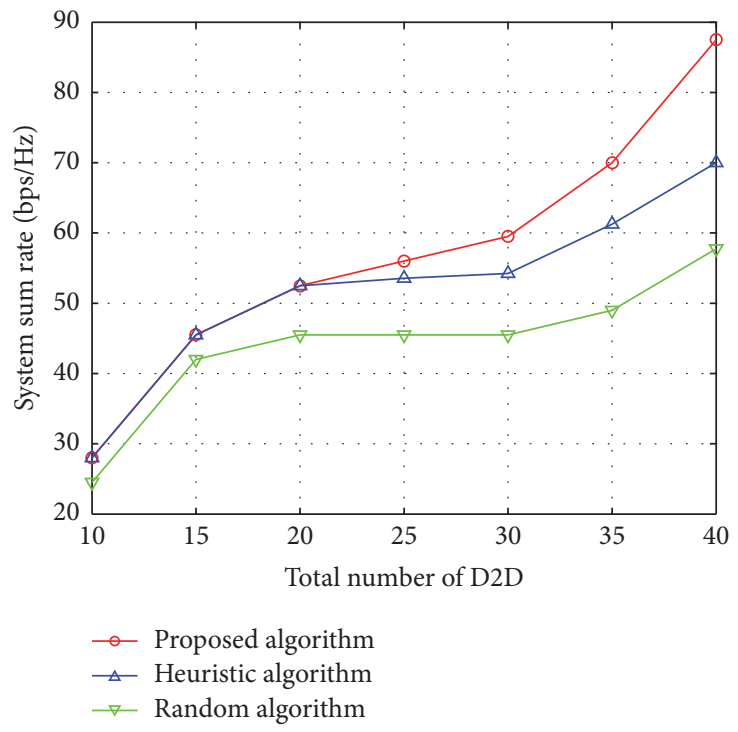

(c) $I_{0}=-130 \mathrm{~dB}$

FIGURE 4: System sum rate with the number of D2D under different interference thresholds.

the random algorithm [21] and the heuristic algorithm [22]; both of them assume that cellular UE can only share resource with a D2D pair. In the random algorithm, a D2D pair shares resource with random cellular UE in the cell, while cellular UE of priority shares resource with a D2D pair which causes the least interference to the eNodeB in the heuristic algorithm.

In Figure 3, the number of D2D pairs in each group is presented. It can be observed that the number of $\mathrm{D} 2 \mathrm{D}$ pairs in the first group is increasing while the number of D2D pairs in the third group is decreasing with the interference threshold value of eNodeB becoming smaller. Since D2D pairs in the first group can only use exclusive resource, some of them cannot access the system when exclusive resource is insufficient. Next, we analyze the performance of the proposed scheme.

The system sum rate with the number of D2D under different interference thresholds can be found in Figure 4. It can be seen that the system sum rate of the proposed algorithm is better than the other two algorithms.

In Figure 5, since the proposed algorithm enables multiple D2D UE devices to share resource with the same cellular UE simultaneously, it achieves a higher network capacity than the other two algorithms. From Figure 5(a), we can 


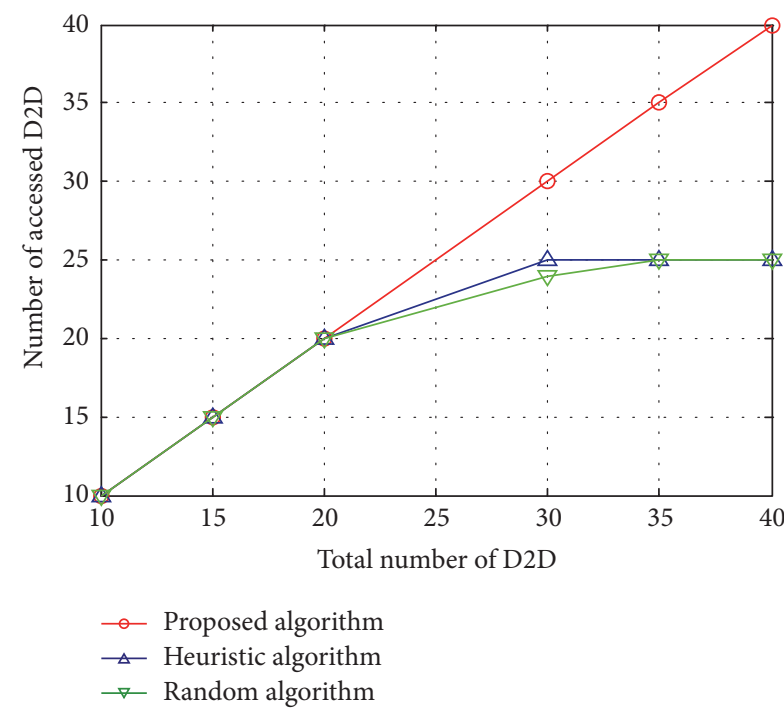

(a) $I_{0}=-100 \mathrm{~dB}$

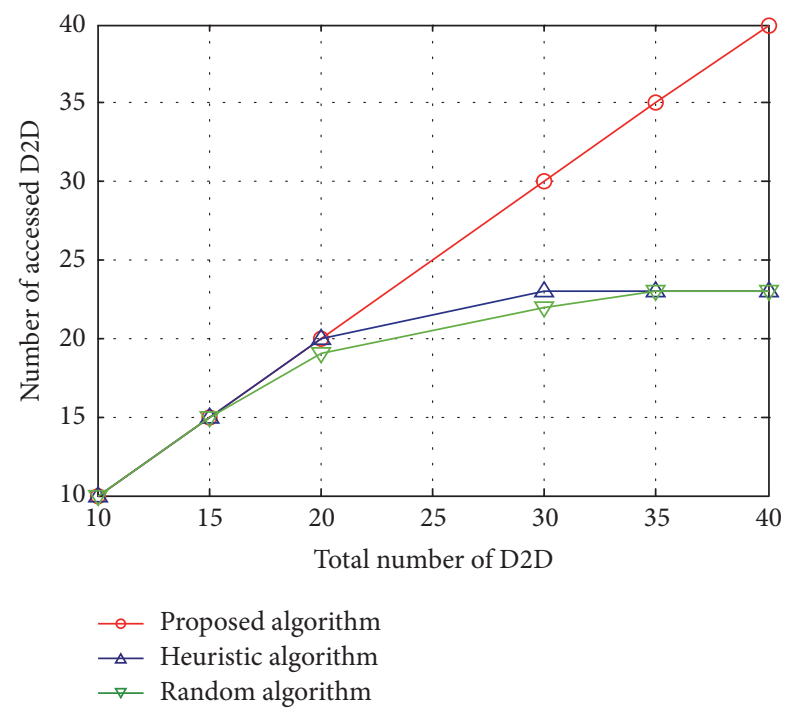

(b) $I_{0}=-120 \mathrm{~dB}$

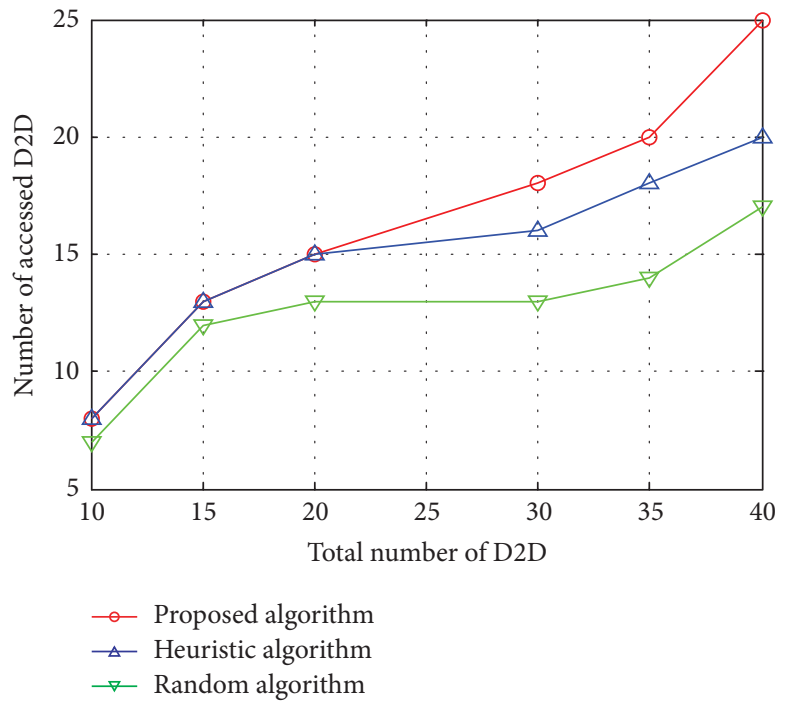

(c) $I_{0}=-130 \mathrm{~dB}$

FIGURE 5: The number of accessed D2D pairs.

see that the maximum number of accessed D2D pairs is 25 which is equal to cellular UE. The reason is that cellular UE can only share resource with a D2D pair in the other two algorithms. At the same time, it can be observed that the number of accessed D2D pairs is decreasing with the interference threshold value of eNodeB becoming smaller. When many D2D pairs belong to the first group and they can only use exclusive limited resources, as a result, some D2D pairs have no resource to use and thus cannot access the system.

The average transmit power of D2D transmitter is presented in Figure 6. It shows that the proposed resource sharing method has a better performance in the aspect of transmit power over the random algorithm and heuristic algorithm. The reason is as follows. In the random algorithm, a D2D pair shares resource with random cellular UE, while cellular UE of priority shares resource with D2D pair which causes the least interference to the eNodeB in the heuristic algorithm. However, in the proposed algorithm, cellular UE devices prefer to share resource with the D2D pairs of smaller transmit power.

At the end, we have compared the proposed method with the methods proposed in [11], as shown in Figures 7 and 8 . As for literatures [16-18], the proposed methods indeed achieve relatively better performance and lay the foundation in the area of the D2D resource sharing. However, it is shown that the CA algorithm in [11] can achieve a relatively higher sum rate compared with our proposed method, while the average transmit power is rather higher than the proposed method in our paper. Hence, our proposed method can be regarded as a 


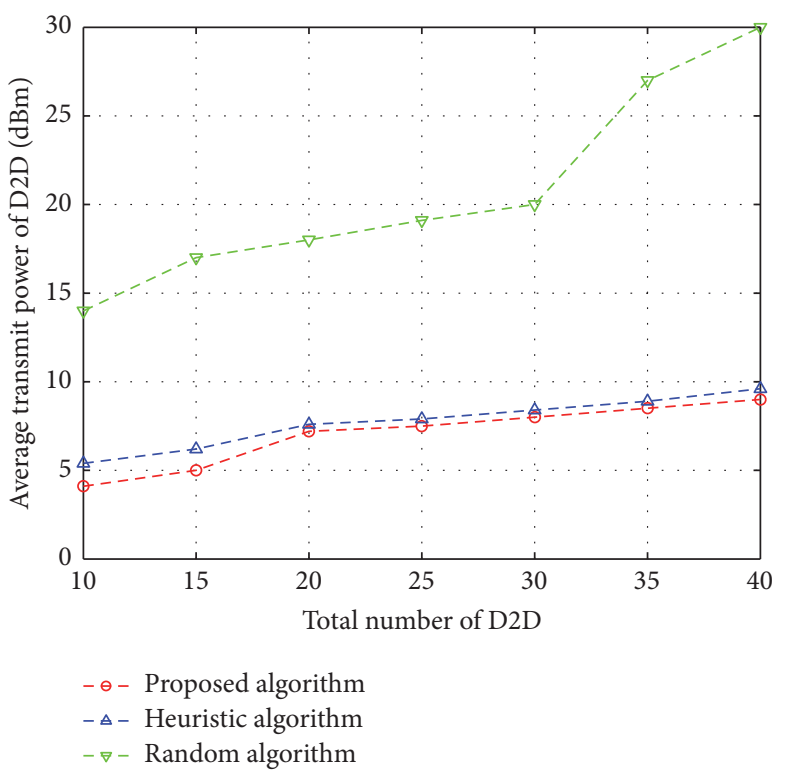

(a) $I_{0}=-100 \mathrm{~dB}$

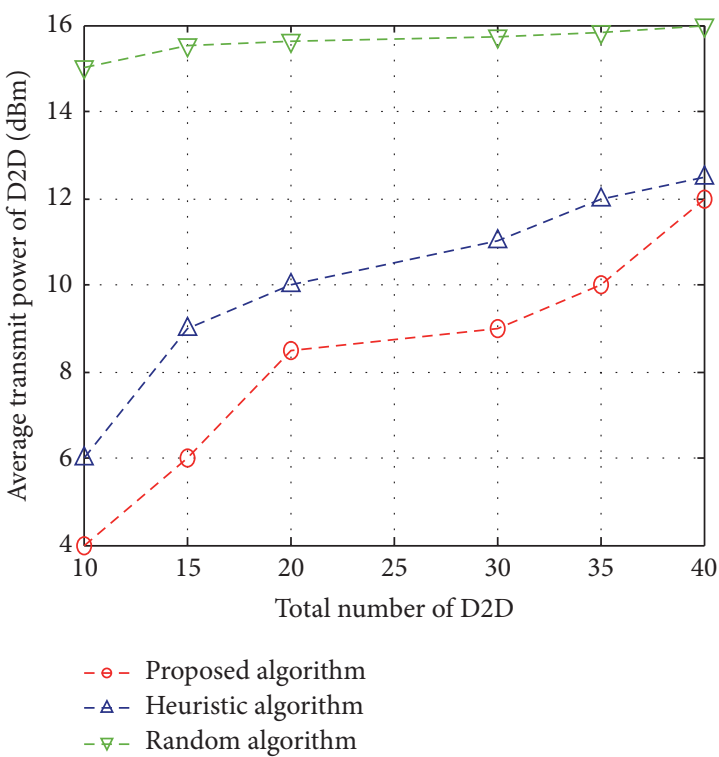

(b) $I_{0}=-120 \mathrm{~dB}$

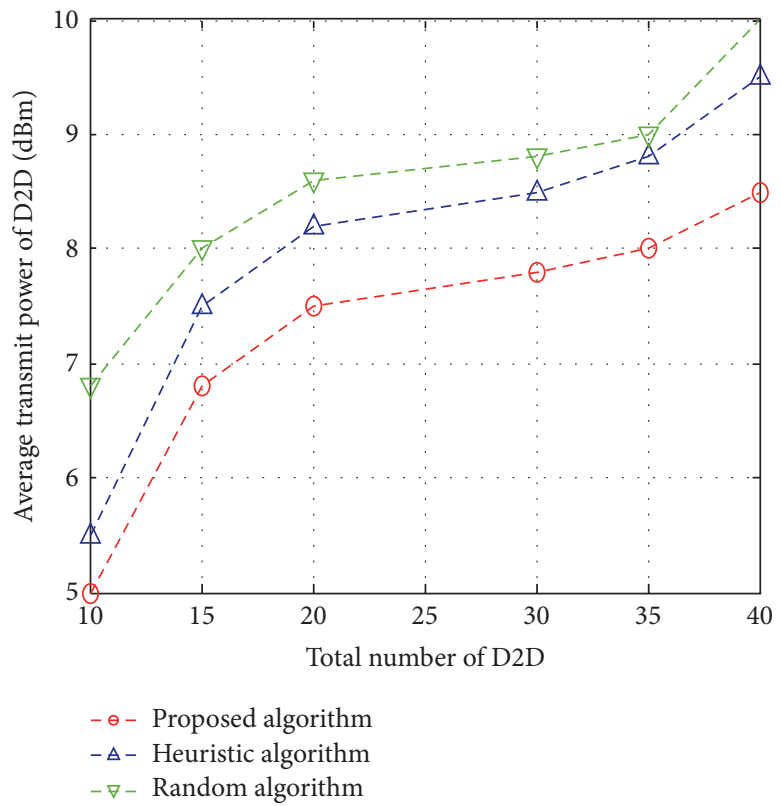

(c) $I_{0}=-130 \mathrm{~dB}$

FIgURE 6: Transmit power of D2D transmitter.

kind of sum rate-power tradeoff scheme and can be used as an alternative in reality.

\section{Conclusion}

In this paper, a mechanism where multiple D2D links share resource with a cellular link in cellular network is proposed. Firstly, a minimum transmit power matrix and a maximum transmit power matrix are constructed, and D2D UE devices are divided into three groups by comparing each element of matrix. We construct a minimum transmit power matrix according to D2D pairs in the second group. By circular searching of the minimum value in matrix and comparing it with the corresponding maximum transmit power, the minimum transmit power values are obtained. Then, we adjust the transmit power of D2D transmitters that belong to the same column, and the D2D pairs share resource with cellular UE by the subscript of the selected minimum transmit power. In order to minimize the transmit power of D2D system, D2D UE devices in the first group and third group share resource with cellular UE based on the transmit power minimization principle. Finally, the simulation results 


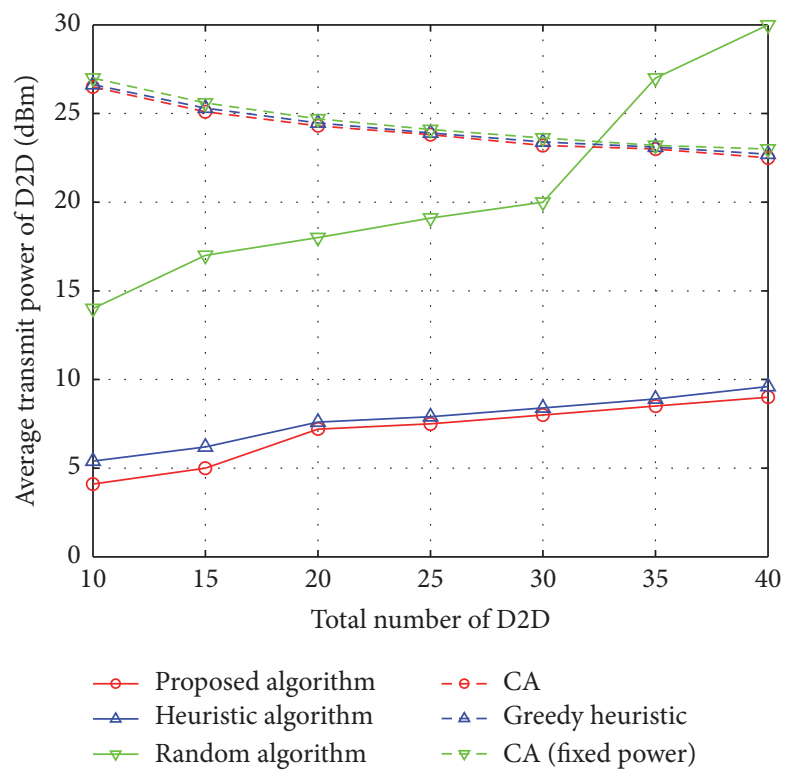

FIGURE 7: Average transmit power with the number of D2D for different methods.

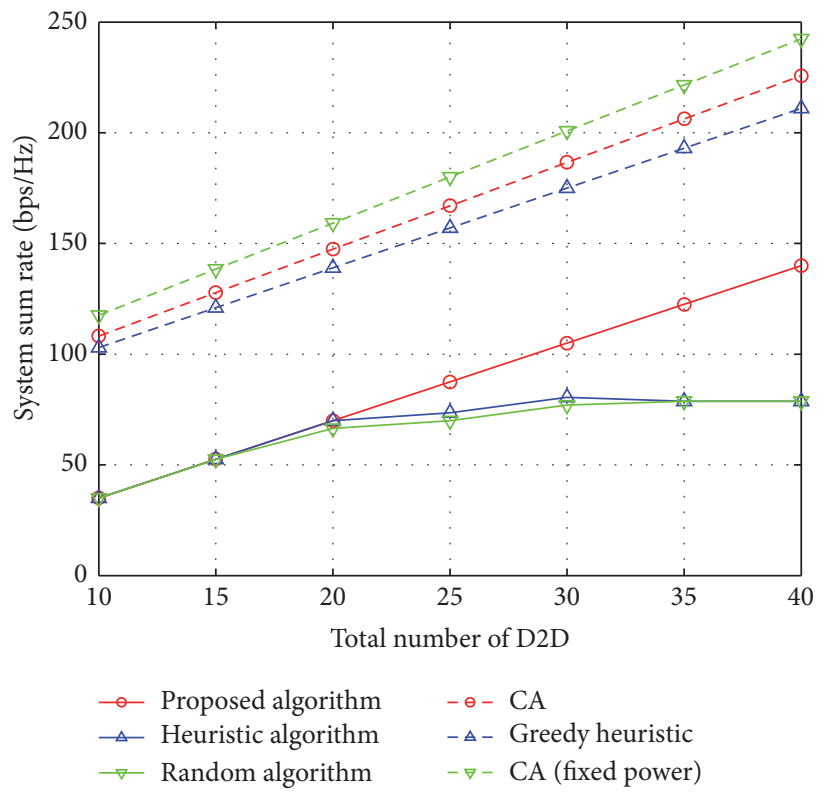

FIGURE 8: System sum rate with the number of D2D for different methods.

show that the proposed policy can achieve a relatively higher network capacity and lower transmit power of D2D system.

\section{Competing Interests}

The authors declare that there are no competing interests regarding the publication of this paper.

\section{Acknowledgments}

This work was partially supported by the National 863 Program under Grant 2015AA01A705 and China Scholarship
Council. The work of H. Li was supported by the National Science Foundation under Grants ECCS-1407679, CNS-1525226, CNS-1525418, and CNS-1543830.

\section{References}

[1] K. Doppler, M. Rinne, C. Wijting, C. B. Ribeiro, and K. Hug, "Device-to-device communication as an underlay to LTEadvanced networks," IEEE Communications Magazine, vol. 47, no. 12, pp. 42-49, 2009.

[2] G. Fodor, E. Dahlman, G. Mildh et al., "Design aspects of network assisted device-to-device communications," IEEE Communications Magazine, vol. 50, no. 3, pp. 170-177, 2012. 
[3] 3GPP, "Feasibility study for proximity services (ProSe) (release 12)," TR 22.803, 2015.

[4] C.-H. Yu, K. Doppler, C. B. Ribeiro, and O. Tirkkonen, "Resource sharing optimization for device-to-device communication underlaying cellular networks," IEEE Transactions on Wireless Communications, vol. 10, no. 8, pp. 2752-2763, 2011.

[5] J. Gu, S. J. Bae, B.-G. Choi, and M. Y. Chung, "Dynamic power control mechanism for interference coordination of deviceto-device communication in cellular networks," in Proceedings of the 3rd International Conference on Ubiquitous and Future Networks (ICUFN '11), pp. 71-75, IEEE, Dalian, China, June 2011.

[6] C.-H. Yu, O. Tirkkonen, K. Doppler, and C. Ribeiro, "On the performance of device-to-device underlay communication with simple power control," in Proceedings of the IEEE 69th Vehicular Technology Conference (VTC Spring '09), pp. 1-5, April 2009.

[7] C.-H. Yu, O. Tirkkonen, K. Doppler, and C. Ribeiro, "Power optimization of device-to-device communication underlaying cellular communication," in Proceedings of the IEEE International Conference on Communications (ICC '09), pp. 1-5, IEEE, Dresden, Germany, June 2009.

[8] P. Jänis, V. Koivunen, Ć. Ribeiro, J. Korhonen, K. Doppler, and K. Hugl, "Interference-aware resource allocation for device-todevice radio underlaying cellular networks," in Proceedings of the IEEE 69th Vehicular Technology Conference (VTC '09), pp. 1-5, IEEE, Barcelona, Spain, April 2009.

[9] T. Peng, Q. Lu, and H. Wang, "Interference avoidance mechanisms in the hybrid cellular and device-to-device systems," in Proceedings of the IEEE 20th International Symposium on PIMRC, pp. 617-621, 2009.

[10] M. Zulhasnine, C. Huang, and A. Srinivasan, "Efficient resource allocation for device-to-device communication underlaying LTE network," in Proceedings of the IEEE 6th International Conference on Wireless and Mobile Computing, Networking and Communications (WiMob '10), pp. 368-375, IEEE, Ontario, Canada, October 2010.

[11] F. Wang, C. Xu, L. Song, and Z. Han, "Energy-efficient resource allocation for device-to-device underlay communication," IEEE Transactions on Wireless Communications, vol. 14, no. 4, pp. 2082-2092, 2015.

[12] X. Lin, J. G. Andrews, A. Ghosh, and R. Ratasuk, "An overview of 3GPP device-to-device proximity services," IEEE Communications Magazine, vol. 52, no. 4, pp. 40-48, 2014.

[13] H. Min, J. Lee, S. Park, and D. Hong, "Capacity enhancement using an interference limited area for device-to-device uplink underlaying cellular networks," IEEE Transactions on Wireless Communications, vol. 10, no. 12, pp. 3995-4000, 2011.

[14] M. Belleschi, G. Fodor, D. D. Penda, A. Pradini, M. Johansson, and A. Abrardo, "Benchmarking Practical RRM Algorithms for D2D Communications in LTE Advanced," Wireless Personal Communications, vol. 82, no. 2, pp. 883-910, 2015.

[15] M. G. S. Rêgo, T. F. Maciel, H. H. M. Barros, F. R. P. Cavalcanti, and G. Fodor, "Performance analysis of power control for device-to-device communication in cellular MIMO systems," in Proceedings of the 2nd International Workshop on Self Organizing Networks (IWSoN '12), August 2012.

[16] R. Zhang, X. Cheng, L. Yang, and B. Jiao, "Interference-aware graph based resource sharing for device-to-device communications underlaying cellular networks," in Proceedings of the IEEE Wireless Communications and Networking Conference (WCNC '13), pp. 140-145, April 2013.
[17] P. Phunchongharn, E. Hossain, and D. Kim, "Resource allocation for device-to-device communications underlaying LTEadvanced networks," IEEE Wireless Communications, vol. 20, no. 4, pp. 91-100, 2013.

[18] J. Wang, D. Zhu, C. Zhao, J. C. F. Li, and M. Lei, "Resource sharing of underlaying device-to-device and uplink cellular communications," IEEE Communications Letters, vol. 17, no. 6, pp. 1148-1151, 2013.

[19] D. H. Lee, K. W. Choi, W. S. Jeon, and D. G. Jeong, “Twostage semi-distributed resource management for device-todevice communication in cellular networks," IEEE Transactions on Wireless Communications, vol. 13, no. 4, pp. 1908-1920, 2014.

[20] 3GPP, "Physical layer aspects for evolved universal terrestrial radio access (UTRA) (release 7)," TS 25.814, 2006.

[21] B. Wang, L. Chen, X. Chen, X. Zhang, and D. Yang, "Resource allocation optimization for device-to-device communication underlaying cellular networks," in Proceedings of the IEEE 73rd Vehicular Technology Conference (VTC '11), pp. 1-6, IEEE, Budapest, Hungary, May 2011.

[22] Y. Xu, R. Yin, T. Han, and G. Yu, "Dynamic resource allocation for Device-to-Device communication underlaying cellular networks," International Journal of Communication Systems, vol. 27, no. 10, pp. 2408-2425, 2014. 

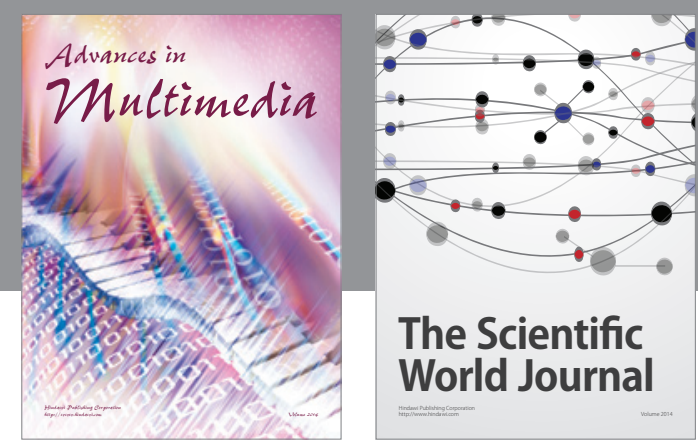

The Scientific World Journal
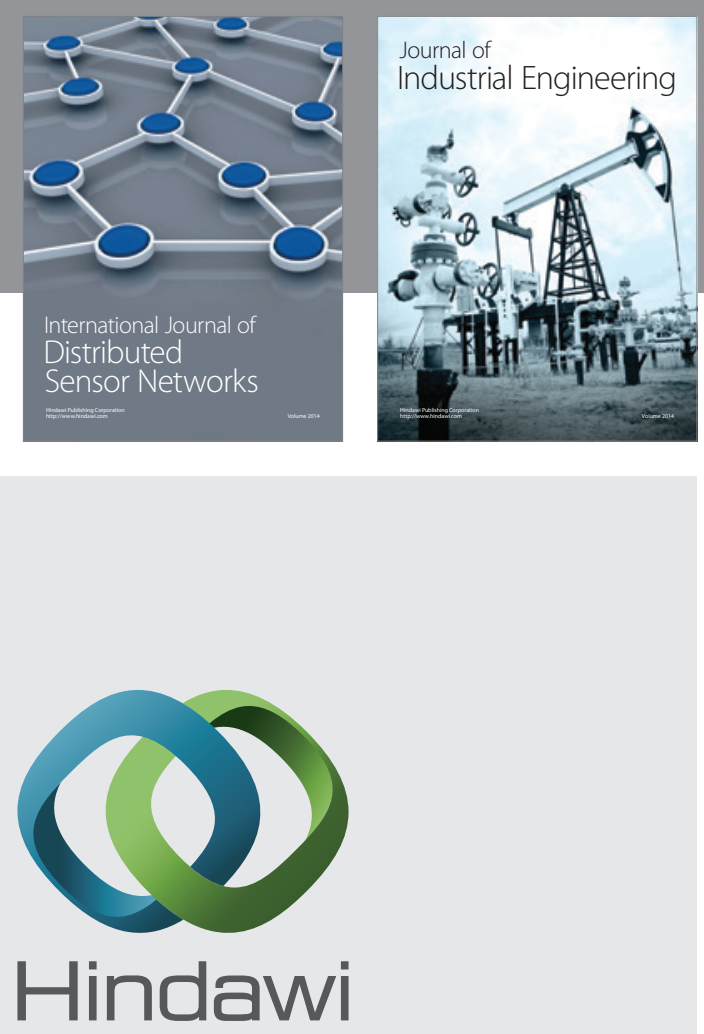

Submit your manuscripts at

http://www.hindawi.com

\section{Computer Networks} and Communications
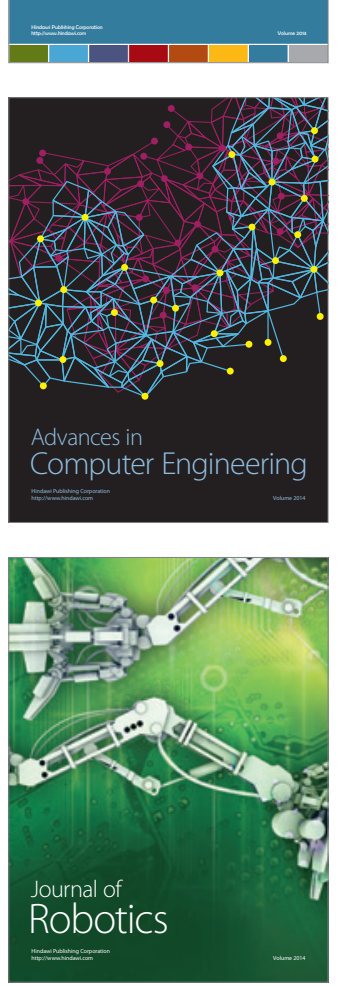
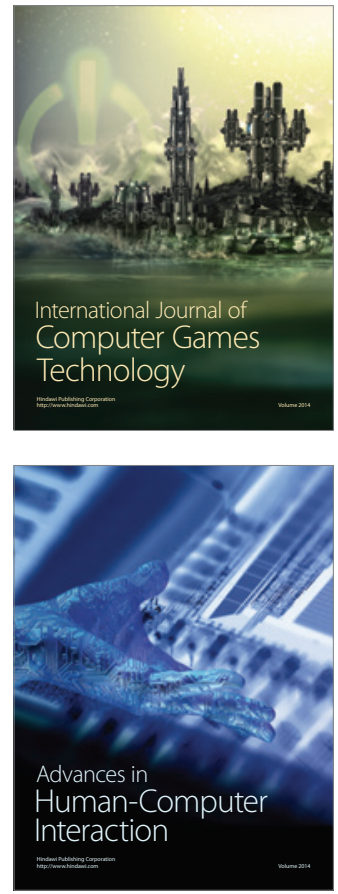
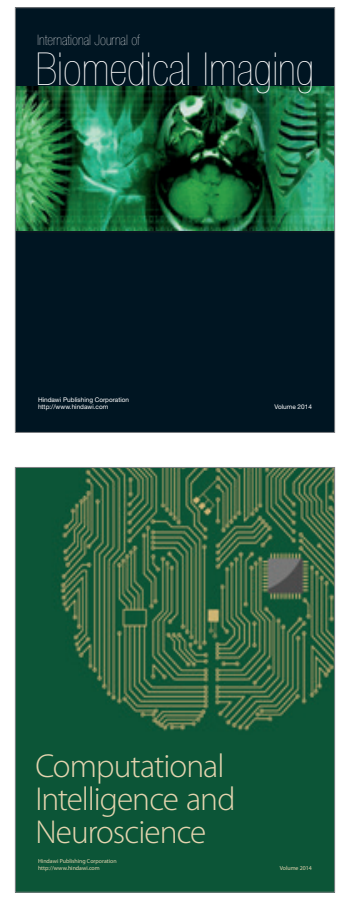
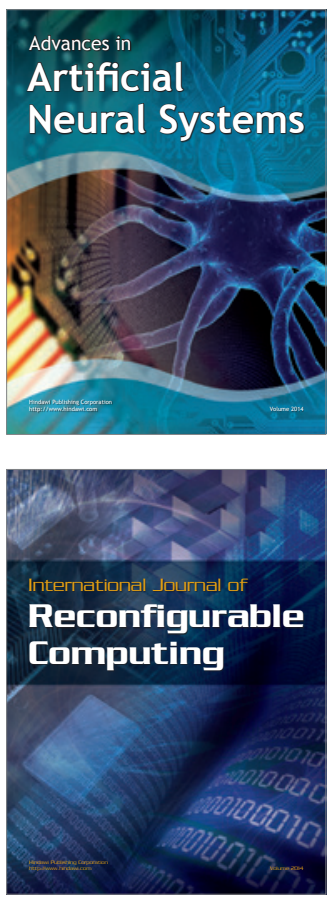
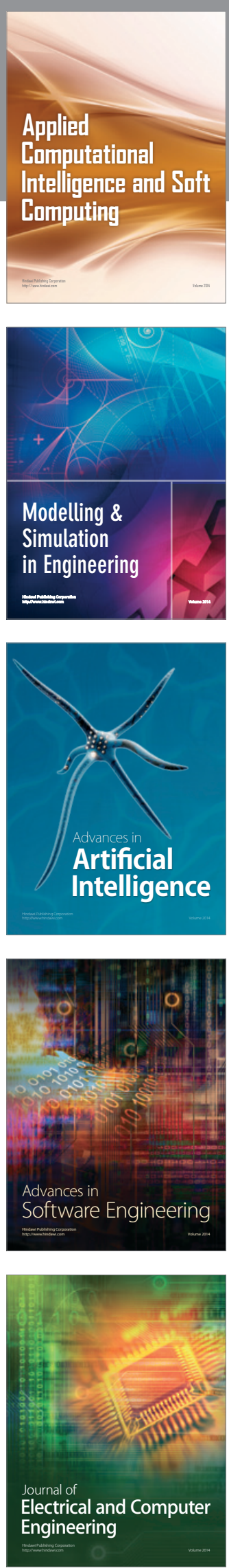